\title{
Non-Thermal Argon Plasma Assisted Antibiotic Therapy: Improving the Efficacy of Ciprofloxacin Against Multi-Drug Resistant P. Aeruginosa Biofilm
}

S. Khosravi

University of Guilan

S. Jafari ( $\nabla$ sjafari@guilan.ac.ir)

University of Guilan

H. Zamani

University of Guilan

M. Nilkar

Ghent University

\section{Research Article}

Keywords: Atmospheric pressure non-thermal plasmas, Argon plasma jet, Ciprofloxacin, Pseudomonas aeruginosa, biofilm

Posted Date: January 27th, 2022

DOI: https://doi.org/10.21203/rs.3.rs-1280279/v1

License: (c) (i) This work is licensed under a Creative Commons Attribution 4.0 International License. Read Full License 


\title{
Non-thermal Argon Plasma Assisted Antibiotic Therapy: Improving the Efficacy of Ciprofloxacin against Multi-Drug Resistant $\boldsymbol{P}$. aeruginosa Biofilm
}

\author{
S. Khosravi ${ }^{1}$, S. Jafari ${ }^{*}, 1$, H. Zamani ${ }^{2}$ and M. Nilkar ${ }^{1,3}$ \\ ${ }^{1}$ Department of Physics, Faculty of Science, University of Guilan, 41335-1914, Rasht, Iran \\ ${ }^{2}$ Department of Biology, Faculty of Science, University of Guilan, 41335-1914, Rasht, Iran \\ ${ }^{3}$ Research Unit Plasma Technology (RUPT), Department of Applied Physics, Faculty of Engineering \& \\ Architecture, Ghent University, Sint-Pietersnieuwstraat 41 B4, 9000 Ghent, Belgium \\ * Corresponding author: sjafari@ guilan.ac.ir
}

\begin{abstract}
Bacterial biofilm formation is regarded as the major cause of infection development, therapeutic failure, and antibiotic resistance. For the first time, the current work aims to characterize the synergistic antibiofilm potential of the atmospheric pressure non-thermal argon plasma and Ciprofloxacin (CIP) against multi-drug resistant Pseudomonas aeruginosa. Bacterial biofilms pre-treated with the non-thermal Ar-plasma, and subsequently treated with CIP antibiotic. Then, bacterial biofilm formation level and cell viability were investigated using the crystal violet staining, MTT, SEM, and fluorescence microscopy. It was found that short-time (a few seconds) pretreating with Ar-plasma not only reduced bacterial biofilm but also significantly decreased the viability of the cells within the biofilm matrix. According to the results, treating with CIP alone reduced bacterial biofilm by $32 \%$, while pretreatment with Ar-plasma for 5, 10, 30, 60, and $90 \mathrm{sec}$ and subsequent antibiotic treatment reduced bacterial biofilm by 76, 79, 81, 75, and $88 \%$, respectively. Besides, the viability of bacterial cells within biofilm matrix reduced to $66 \%$ in CIP treated cells, while, pretreating with Ar-plasma for 5, 10, 30, 60, and $90 \mathrm{sec}$ significantly decreased cell viability to $28,25,21,23$, and $15 \%$, respectively. This concept opens a promising approach for the treatment of drug-resistant bacteria for decontamination of the heat-sensitive materials and human tissues based on the combination of atmospheric pressure non-thermal Ar-plasma and antibiotics.
\end{abstract}

Keywords: Atmospheric pressure non-thermal plasmas; Argon plasma jet; Ciprofloxacin; Pseudomonas aeruginosa; biofilm. 
Bacterial cells are commonly found in biofilm form, which is a bacterial community encased in extracellular polymeric substances (EPS). Biofilm formation by pathogenic bacteria is a major challenge for the eradication of bacterial infection and contamination [1]. Pseudomonas aeruginosa is a Gram-negative opportunistic bacterial pathogen that is responsible for a variety of human infections and contamination of abiotic materials used in medicine [2]. Different chemotherapy strategies could be used to combat $P$. aeruginosa including, using biocides, chelators, and antibiotics [3]. P. aeruginosa is well known for its low membrane permeability and active efflux pumps that aim to reduce the intracellular concentration of antimicrobials [4]. In addition, biofilm formation by $P$. aeruginosa is considered the main cause of multiple drug resistance and infection development. Bacteria enclosed in biofilm show significantly higher resistance to antimicrobials due to the low metabolic rate, slow diffusion of antibiotics into biofilm matrix, and facilitated exchange of drug resistance gene [5]. Therefore, eradication of bacterial biofilm could increase bacterial susceptibility to antimicrobial agents which could facilitate bacterial decontamination and infection control. Several approaches have been suggested to overcome drug-resistant bacteria, including the combined drug therapy [6], use of nanoparticles [7], and also atmospheric-pressure non-thermal plasma treatment [8]. In the last decade, atmospheric pressure non-thermal discharges were studied for the treatment of skin and wound infections, treatment of cancer, sterilization, etc. [9].

Nowadays, the use of atmospheric-pressure plasmas jets (APPJs) is a fascinating approach which is typically operated at room temperature and thus, could be directly applied on heat-sensitive materials and human tissues $[10,11]$. APPJs benefit from relatively low operational cost, usage of non-toxic gases, and the lack of harmful residues [12]. The ability to produce chemically rich plasmas at ambient temperature and their capability to improve the efficacy of biological processes has led to the emergence of the nascent field of plasma medicine [13]. The influence of non-thermal plasma depends on the synergistic action of the generation of chemically reactive species including reactive oxygen and nitrogen species like $\mathrm{OH}, \mathrm{H}_{2} \mathrm{O}_{2}, \mathrm{O}_{3}, \mathrm{NO}$, and $\mathrm{NO}_{2}$, charged particles, and UV radiation [14]. These reactive species can give rise to oxidative stress and peroxidation of membrane lipids, damage bacterial DNA, and cause irreversible changes in the native structure of enzymes or membrane permeability [15]. In recent years, the combination of non-thermal plasma with antimicrobial agents against bacterial biofilm has received considerable interest. Gupta et al. [16] investigated the effect of non-thermal plasma in 
combination with chlorhexidine digluconate $(\mathrm{CHX})$ on $P$. aeruginosa biofilms formed on titanium surface and found complete biofilm sterilization. Guo et al. [17] evaluated the effect of plasma pre-treatment on methicillin-resistant Staphylococcus aureus (MRSA) biofilms further treated with antibiotics such as tetracycline, gentamycin, clindamycin, chloramphenicol, ciprofloxacin, rifampicin, and vancomycin. They found that the pre-treatment of MRSA with plasma promoted the elimination of persister cells and increased antibiotic susceptibility. Paldrychova' et al. [18] employed a combination of some antibiotics with air-based plasma treatment to enhance antibiotics action against mature $P$. aeruginosa biofilms formed on Ti-6Al$4 \mathrm{~V}$ alloy. They reported that using $0.25 h$ non-thermal air-based plasma treatment increases the influence of relatively low concentrations of antibiotics. Recently, Muraca et al. [19] investigated the improvement of the antimicrobial activity of CIP through the lipid nanoencapsulation and air-based plasma on $P$. aeruginosa biofilms. A decrement in the minimum inhibitory concentration was reported for biofilms exposed to plasma and free ciprofloxacin, and for biofilms treated with nanoparticles.

In this Letter, the synergistic antimicrobial effects of atmospheric-pressure non-thermal argon plasma and ciprofloxacin (CIP) against multi-drug resistant (MDR) P. aeruginosa biofilms were investigated in vitro. The results revealed that the combination of short-time Ar-plasma pretreatment and CIP significantly enhanced antimicrobial efficiency of the antibiotic which could be associated with the increased permeability of bacterial membrane lipid following the generated oxidative stress, and induced irreversible changes in the structure of bacterial proteins. The schematic of the atmospheric-pressure non-thermal argon plasma jet and CIP treatments on the $P$. aeruginosa biofilm is shown in Fig. 1. As seen, at the center of a quartz capillary of Arplasma jet, a pin-type steel electrode is mounted. Besides, a ring cooper electrode is set as a grounded electrode, and this apparatus is tightly wrapped in a dielectric shell. In the continuous working mode, an AC high-voltage supply provided $\mathrm{Vp}-\mathrm{p}$ of $9 \mathrm{kV}$ at a frequency of $22 \mathrm{kHz}$ to the pin-type electrode. Argon was the working gas and the typical flow rate was relatively low magnitude, i.e., 1.8 standard liter per minute $(\mathrm{s} / \mathrm{m})$. The ignited plasma jet has a length of up to 8 $\mathrm{mm}$. The distance between the $P$. aeruginosa biofilms and plasma jet nozzle was $8 \mathrm{~mm}$. 




FIG. 1. Schematic representation of the atmospheric-pressure non-thermal argon plasma jet and Ciprofloxacin treatments on the $P$. aeruginosa biofilms.

Fresh culture of MDR P. aeruginosa strains (isolated from clinical specimens) was prepared in tryptic soy broth (TSB) medium, and their antibiotic resistance pattern was determined and the strains that showed resistance to at least three classes of antibiotics were considered as MDR strains (data not shown). Then, bacterial biofilm was prepared in 6-well polystyrene plates. In brief, $100 \mu \mathrm{L}$ of a fresh bacterial strain with a population of $1.5 \times 10^{6} \mathrm{cfu} / \mathrm{mL}$ was inoculated in the TSB medium. The plate was incubated for $72 h$ at $37^{\circ} \mathrm{C}$ without shaking. After the formation of bacterial biofilm, the wells were washed using sterile physiological saline to remove loosely adherent and unattached cells. Then, four treatment groups were designed, including $a$ ) untreated control cells, $b)$ treatment with CIP $(16 \mu \mathrm{g} / \mathrm{mL}), c)$ Ar-plasma jet treatment, $d)$ pre-treatment with Ar-plasma jet and subsequent treatment with CIP $(16 \mu \mathrm{g} / \mathrm{mL})$. Exposure to Ar-plasma jet was 
performed in a range of 5-90 sec $(5 \mathrm{sec}, 10 \mathrm{sec}, 30 \mathrm{sec}, 60 \mathrm{sec}$, and $90 \mathrm{sec})$. The respecting plates were treated with CIP at $16 \mu \mathrm{g} / \mathrm{mL}$, as a sub-inhibitory concentration. The sub-inhibitory concentration of the drug was selected based on the minimum inhibitory concentration assay (data not shown). The plates were further incubated for $18 \mathrm{~h}$ at $37^{\circ} \mathrm{C}$ and then, subjected to the subsequent analysis, including biofilm quantification, determination of bacterial survival (MTT assay), as well as biofilm visualization using fluorescent microscopy and scanning electron microscopy (SEM).

The MTT assay is a colorimetric assay which is based on the conversion of 3-(4,5dimethylthiazol-2-yl)-2,5-diphenyl-2H-tetrazolium bromide (MTT) into formazan crystals by living cells. In brief, $200 \mu L$ of $10 \%$ MTT (Sigma-Aldrich) solution was added to each well. The plate was incubated for $3 h$ at $37^{\circ} \mathrm{C}$ and then, $200 \mu \mathrm{L}$ of DMSO was added to solubilize formazan crystals. After shaking for $20 \mathrm{~min}$ the optical density (OD) of each well was measured at $540 \mathrm{~nm}$. The cell survival is calculated as follows [20],

Survival rate $(\%)=(\mathrm{OD}$ of treated well $) /($ mean OD of control wells $) \times 100$

In addition, biofilm level in the wells from different treatment groups was quantified using the crystal violet staining assay. The $\mathrm{OD}_{540}$ was recorded using a plate reader (Bio-Rad, USA). The biofilm inhibition was calculated using the following formula [21],

Biofilm inhibition $(\%)=[($ Control OD _Test OD $) /$ Control OD $] \times 100$

Non-thermal Ar-plasma-assisted antibiofilm and antibacterial effect of CIP against MDR $P$. aeruginosa mature biofilm was demonstrated by the MTT and crystal violet assay. The results are summarized in Figs. 2 and 3. According to the results, treating bacterial biofilm with CIP alone, reduced cell viability to $66 \%$, compared with the untreated cells. Moreover, Ar-plasma exposure reduced cell viability to 60 and $40 \%$ after 5 and $90 \mathrm{sec}$ exposure. It was found that Arplasma-assisted antibiotic therapy reduced the viability of bacterial cells in a range of 72 to $85 \%$, depending on the duration of Ar-plasma pre-treatment (5-90 sec). Comparing the treatment groups, our result showed a significant decrease in the viability of bacterial cells in mature biofilm after pre-treatment with Ar-plasma and subsequent treatment with CIP, at sub-inhibitory concentration. Surprisingly, pre-treatment with Ar-plasma, even for 5 sec, dramatically 
decreased the viable $P$. aeruginosa population compared with the cells that were only treated with CIP alone.

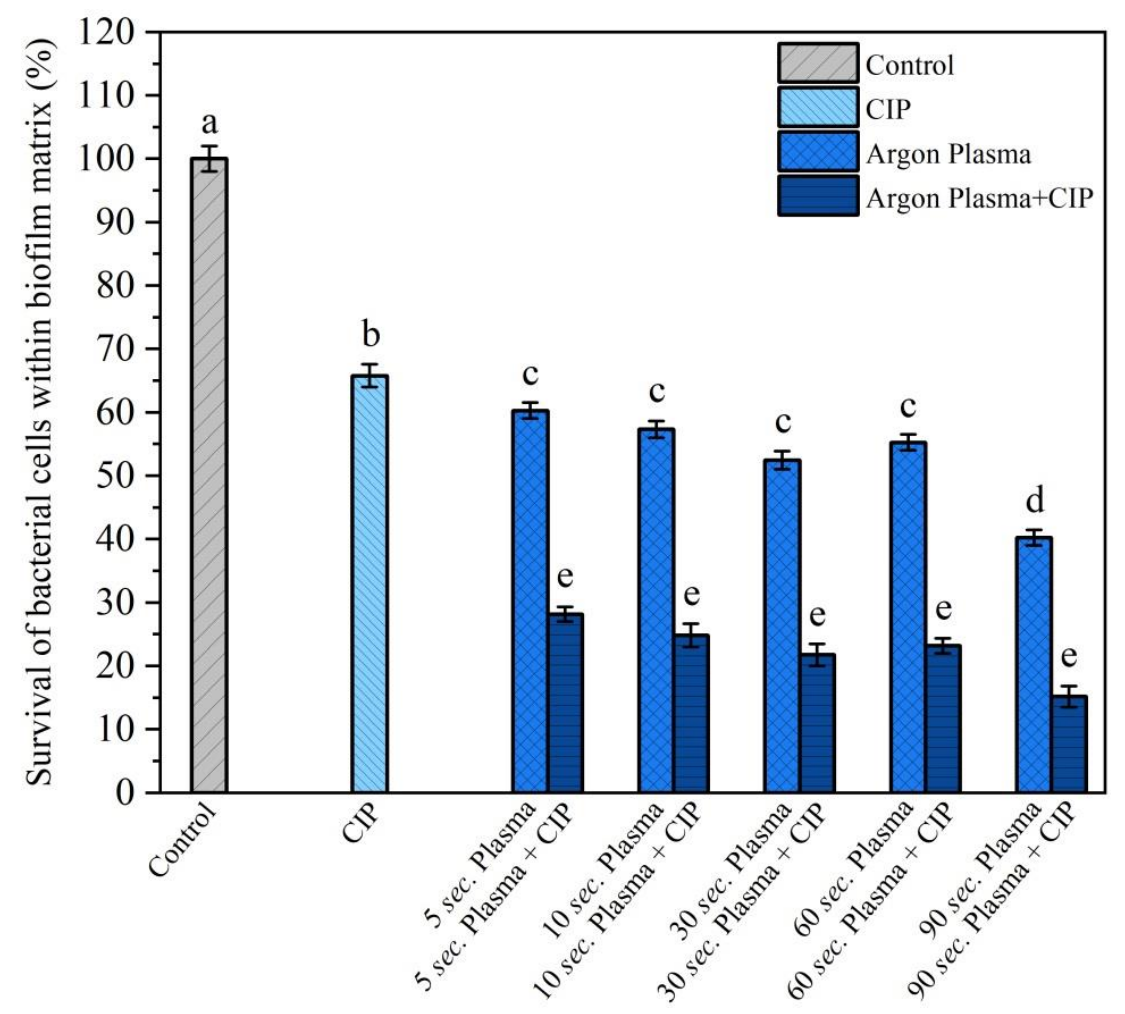

FIG. 2. MTT assay of biofilm survival percentage in the combination of argon plasma with CIP against $P$. aeruginosa biofilm.

Quantification of bacterial biofilms from different treatment groups was performed using the crystal violet staining assay. This assay is dependent on the staining of the attached cells on the examined surfaces followed by the quantification of the absorbed dye. The results revealed that treating $P$. aeruginosa with CIP alone reduced bacterial biofilm to $67 \%$, which was significantly lower than untreated control wells. In addition, pre-treatment of the cells with Ar-plasma followed by the treatment with CIP significantly reduced the biofilm level of $P$. aeruginosa. We found that exposure to Ar-plasma for even $5 \mathrm{sec}$ prior to treating with CIP significantly decreased biofilm level to $24.14 \%$ compared with the biofilms that were only treated with CIP 
alone (67.78\%). In addition, longer pre-treatment time (up to $90 \mathrm{sec}$ ) with Ar-plasma reduced bacterial biofilm up to $12.16 \%$.

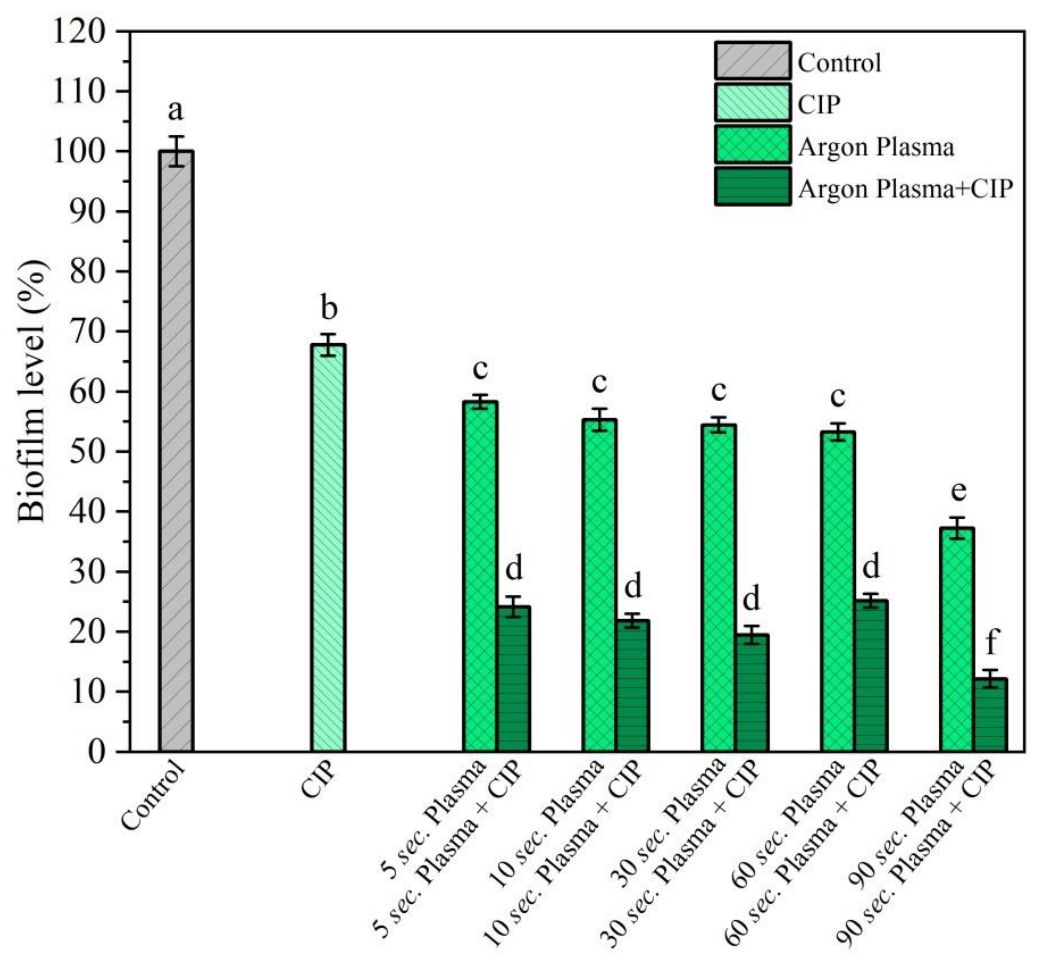

FIG. 3. Crystal violet staining biofilm survival percentage in the combination of argon plasma with CIP against $P$. aeruginosa biofilm.

To confirm the results obtained from the MTT and crystal violet assays, fluorescence microscopy using SYTO®13 dye and SEM were performed. The SEM images revealed biofilm disruption in the Ar-plasma exposed biofilms. It was found that, although treating bacterial biofilm with CIP alone did not considerably reduce bacterial cells within the preformed biofilm, pre-treatment with Ar-plasma efficiently reduced the number of attached bacterial cells in the biofilm matrix. As could be observed in Fig. 4, the longer pre-treatment time with Ar-plasma caused a greater bacterial cell lysis and therefore, more efficient biofilm eradication. In other words, in the wells with Ar-plasma pre-treatment for $30 \mathrm{sec}$ and longer, intact bacterial cells are hardly observed, suggesting complete rapture of the bacterial cell wall and complete biofilm eradication. In addition, fluorescence microscopy with staining by SYTO®13 dye was used to visualize the biofilms, on the basis of the affinity of the fluorescent dye for binding to the nucleic acids of bacterial cells, and also extracellular DNA molecules that are commonly found in the biofilm 
matrix. The results revealed that the untreated mature biofilm of MDR $P$. aeruginosa was very robust and the entire surface of the control wells was completely covered by bacterial biofilm, which could be visualized by the presence of a large number of bright green dots (Fig. 5). Fluorescent microscopy of the CIP treated wells indicated a decrease in the biofilm coverage (around 35\% reduction); however, a large area of the surface of the wells was still covered with the bacterial biofilm. Although treating the biofilms with Ar-plasma alone reduced bacterial biofilm, some bacterial cells were still attached to the surface indicating the incomplete eradication of bacterial biofilm. Interestingly, the results revealed that pre-treatment of $P$. aeruginosa biofilm with Ar-plasma and subsequent treating with Ciprofloxacin remarkably reduced the frequency of the bright green dots, indicating the major eradication of bacterial biofilm.



$5 \mu \mathrm{m}=$


$20 \mu \mathrm{m}$
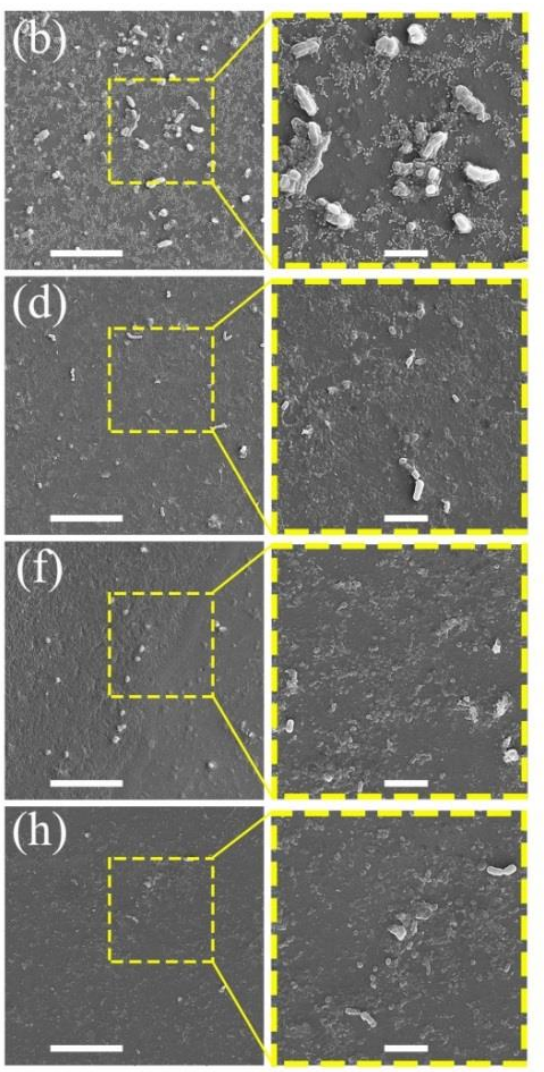

FIG. 4. SEM images of $P$. aeruginosa mature biofilm treated with CIP alone or combined with non-thermal Arplasma pre-treatment at different exposure times: (a) Untreated control, (b) CIP treatment, (c) 5 sec Ar-plasma, (d) 5 sec Ar-plasma combined with CIP, (e) $30 \mathrm{sec}$ Ar -plasma, (f) $30 \mathrm{sec}$ Ar-plasma combined with CIP, (g) 90 sec Ar plasma, and (h) 90 sec Ar-plasma combined with CIP. 
Control



$90 \mathrm{sec}$. Plasma
CPR Antibiotic

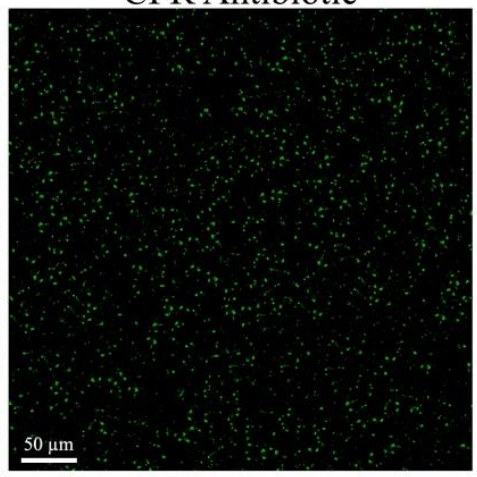

90 sec. Plasma + Antibiotic
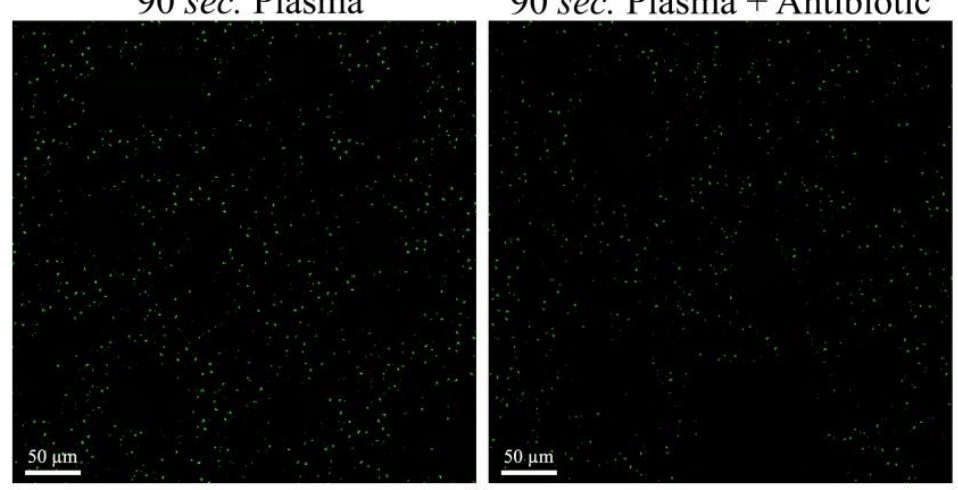

FIG. 5. Fluorescence microscopy images of $P$. aeruginosa mature biofilm treated with Ciprofloxacin (CIP) alone or combined with Ar-plasma pre-treatment. (a) Untreated control, (b) CIP treatment, (c) 90 sec Ar-plasma, and (d) 90 sec Ar-plasma combined with CIP.

Biofilm formation is considered a major prerequisite of infection establishment and development of infectious diseases. Bacterial cells are mainly found in biofilm form, which could protect them against the host immune system and also antimicrobials. Due to the decreased penetration of antibiotics, bacterial biofilms are regarded as the major cause of antibiotic resistance and therapeutic failure [22]. Evaluation of the metabolic activity of bacterial cells (as a survival indicator) revealed that primary exposure of bacterial biofilm with Ar-plasma significantly enhanced bactericidal activity of subsequent antibiotic therapy. While treating bacterial biofilm with CIP alone, did not considerably reduce bacterial survival, which could be associated with the low penetration of the drug into the biofilm matrix. Quantification of bacterial biofilm also confirmed the biofilm disruption following Ar-plasma exposure, confirming the MTT results. Further, the SEM and fluorescent microscopy assays obviously revealed that pre-treatment with Ar-plasma and subsequent treatment with ciprofloxacin could efficiently eradicate bacterial biofilm. As described above, the antibacterial activity of non-thermal plasma is mainly 
associated with the generation of free oxygen and nitrogen radicals with could provide oxidative stress. The generated reactive radical molecules interact with bacterial cell components that could damage bacterial cell envelopes, including cell membrane and cell wall and cause increased cell permeability $[23,24]$. In addition, the generated radicals are able to disrupt the biofilm matrix by degradation of the extracellular polymeric substances, including polysaccharides, glycoproteins, DNA, etc., which could enhance the permeability of antibiotic into the bacterial biofilm. Ciprofloxacin is a commonly prescribed antibiotic with bactericidal activity through the inhibition of DNA replication. It seems that pre-treatment with Ar-plasma could increase the permeability of bacterial cells and also penetration of antibiotic into the biofilm matrix and facilitates antibiotic diffusion into bacterial cytoplasm, which could improve the efficacy of CIP against $P$. aeruginosa biofilm.

In summary, for the first time, we investigated the effect of non-thermal Ar-plasma pre-treatment on the efficacy of CIP antibiotic against multi-drug resistant $P$. aeruginosa biofilm, in vitro. The antimicrobial effects of the argon plasma jet operated by AC power supply driven at $9 \mathrm{kV}$ and 22 $\mathrm{kHz}$. Non-thermal plasma jets have received considerable interest in plasma-medicine due to the effective and rapid inactivation, non-toxic residues, and cost effectuality. For evaluating the biofilm removal efficiency, bacterial biofilms were exposed to the short-time argon plasma and subsequently treated with CIP. The Ar-plasma jet assisted antibiotic therapy significantly improved the efficacy of CIP and efficiently eradicated bacterial biofilm in a time-dependent manner. According to the results, treating with CIP alone reduced bacterial biofilm by $32 \%$, while pretreatment with Ar-plasma for 5, 10, 30, 60, and $90 \mathrm{sec}$ and subsequent antibiotic treatment reduced bacterial biofilm by $76,79,81,75$, and $88 \%$, respectively. The synergism of Ar-plasma pretreatment with CIP could be associated with the generation of free radical molecules that may increase drug penetration into the biofilm matrix and also increased permeability of antibiotic into bacterial cells. This work revealed that the combination of shorttime Ar-plasma jet pre-treatment and subsequent antibiotic therapy could be a promising approach to combat drug-resistant $P$. aeruginosa biofilms, which could be used in clinical applications after in-vivo experiments. 
Author's Contributions S. Khosravi: investigation, collected and analyzed the data, writing — original draft. S. Jafari: methodology, supervision, conceived and designed the experiments, review, and editing. H. Zamani: methodology, supervision, conceived and designed the experiments, review, and editing. M. Nilkar: methodology, supervision, conceived and designed the experiments, review, and editing. All authors discussed the results and commented on the paper.

Competing interests The authors declare no competing interests.

Ethics Approval This article does not contain any studies with human participants performed by any of the authors.

Data Availability The data that supports the findings of this study are available within the article.

\section{References}

[1] M. Jansen, A. Wahida, S. Latz, A. Krüttgen, H. Häfner, E.M. Buhl, et al., Scientific Rep. 8 (1), 1(2018)

[2] F. Khan, D. T. N. Pham, S. F. Oloketuyi and Y. M. Kim, Appl. Microb. Biotech., 104, 33 (2020).

[3] P.K. Taylor, A. T.Y. Yeung, R. E.W. Hancock, J. Biotech., 191, 121 (2014).

[4] E. Z. Reyes-Fernández, Sh. Schuldiner, Sci. Rep., 10, 9954 (2020).

[5] A. Salehzadeh, H. Zamani, M.K. Langeroudi, A. Mirzaie, Iranian J. Basic Medical Sci., 19(12), 1325 (2016).

[6] N. Vasan, J. Baselga and D. M. Hyman, Nature, 575, 299 (2019).

[7] V. Garofano, L. Stafford, B. Despax, R. Clergereaux, and K. Makasheva, Appl. Phys. Lett., 107, 183104 (2015).

[8] M. Laroussi, X. Lu, M. Keidar, J. Appl. Phys. 122 (2), 020901 (2017).

[9] G. Fridman, G. Friedman, A. Gutsol, A.B. Shekhter, V.N. Vasilets, A. Fridman, Plasma proc. polymers 5 (6), 503-533 (2008).

[10] T. V. Woedtke, S. Emmert, H. R. Metelmann, S. Rupf, K. D. Weltmannn, Phys. plasmas, 27, 070601 (2020).

[11] M. Nilkar, F.E. Ghodsi, S. Jafari, D. Thiry, R. Snyders, J. Alloys and Compounds, 853, 157298 (2021).

[12] M. Keidar, K.D. Weltmann, S. Macheret, J. Appl. Phys. 130 (8), 080401 (2021).

[13] J. Julák, E. Vaňková, M. Válková, P. Kašparová, J. Masák, V. Scholtz, Folia Microbiologica, 65, 863 (2020).

[14] X. Zhang, D. Liu, R. Zhou, Y. Song, Y. Sun, Q. Zhang, J. Niu, H. Fan, and S. Yang, Appl. Phys. Lett. 104, 043702 (2014).

[15] P. Bourke, D. Ziuzina, L. Han, P.J. Cullen, B.F. Gilmore, J. Appl. microbiology 123 (2), 308-324 (2017).

[16] T. T. Gupta, S. B. Karki, J. S. Matson, D. J. Gehling, and H. Ayan, Biomed. Res. Inter., 1-11 (2017).

[17] L. Guo, R. Xu, Y. Zhao, D. Liu, Z. Liu, X. Wang, H. Chen and M. G. Kong, Front. Microbiology, 9:573, 1 (2018).

[18] M. Paldrychova, E. Vankova, P. Kasparova, E. Sembolova, O. Matatkova, J. Masak, V. Scholtz, and J. Julak, World J. Microb. Biotech., 36, 108 (2020).

[19] G.S. Muraca, J. Soler-Arango, G.R. Castro, G.A. Islan, G. Brelles-Mariño, J. of Drug Delivery Sci. Tech., 102644 (2021). 
[20] S. Khosravi, S. Jafari, H. Zamani, M. Nilkar, Appl. Biochem. Biotech., 193, 3641 (2021).

[21] H.T. Mangoudehi, H. Zamani, S. S. Shahangian and L. Mirzanejad, World J. Microb. Biotech., 36, 1-9 (2020).

[22] L. Dai, T.Q. Wu, Y.S. Xiong, H.B. Ni, Y. Ding, W.C. Zhang, \& J. Yu, Life sciences, 237, 116947 (2019).

[23] X. Wang, X. Zhao, Antimicrob. agents chemo, 53(4), 1395 (2009).

[24] X. Zhao, Y. Hong, K. Drlica, J. of Antimicrb. Chemo., 70(3), 639 (2015). 